Article

\title{
Towards Sustainable Public Health Surveillance in India: Using Routinely Collected Electronic Emergency Medical Service Data for Early Warning of Infectious Diseases
}

\author{
Eva Pilot ${ }^{1, *}$, Ramana Roa ${ }^{2}$, Biranchi Jena ${ }^{3}$, Boris Kauhl ${ }^{1}$, Thomas Krafft ${ }^{1}$ and GVS Murthy ${ }^{4,5}$ \\ 1 Department of Health, Ethics and Society, School of Public Health and Primary Care (CAPHRI), \\ Faculty of Health, Medicine and Life Sciences, Maastricht University, 6229HA Maastricht, The Netherlands; \\ boris.kauhl@maastrichtuniversity.nl (B.K.); thomas.krafft@maastrichtuniversity.nl (T.K.) \\ 2 GVK Emergency Management Research Institute, Secunderabad 500078, India; ramanarao_gv@emri.in \\ 3 Indian Institute of Health Management Research, Bangalore 560105, India; jena@iihmr.org \\ 4 Public Health Foundation India, Indian Institute of Public Health Hyderabad, Telangana 500033, India; \\ murthy.gvs@iiphh.org or GVS.Murthy@lshtm.ac.uk \\ 5 London School of Hygiene and Tropical Medicine, London WC1E7HT, UK \\ * Correspondence: eva.pilot@maastrichtuniversity.nl; Tel.: +31-620-311-075
}

Academic Editors: Mark Rosenberg, Wuyi Wang, Thomas Krafft, Linsheng Yang and Yang Cheng Received: 26 February 2017; Accepted: 7 April 2017; Published: 13 April 2017

\begin{abstract}
Infectious disease surveillance, timely detection and early warning of outbreaks present a complex challenge to health authorities in India. Approaches based on the use of unexplored data sources, like emergency medical services (EMS) data, can contribute to the further advancement of public health surveillance capacities in India and support and strengthen the Integrated Disease Surveillance Programme (IDSP) strategy. This research followed a mixed method approach including a series of semi-structured interviews and fever data analysis of the EMS operating dispatch system in Andra Pradesh, India. In this paper, we explore whether routinely collected EMS health data can improve sustainable infectious disease surveillance and early warning capacity. The result highlights the need for improved surveillance systems for early warning of infectious diseases in India. The data availability at the EMS dispatch centre includes patient data and spatial information and can be used for near real-time analysis. Routine data relevant for health surveillance can be extracted to provide timely health information that supplements and enhances more traditional surveillance mechanisms and thus provides a cost-efficient, near real-time early warning system for the operating states. The designed intervention is sustainable and can improve infectious disease surveillance to potentially help the government officials to appropriately prioritize timely interventions to prevent infectious disease spread.
\end{abstract}

Keywords: early warning; surveillance; EMS; infectious disease; information system research; India; syndromic surveillance; International Health Regulations; dengue; AUF

\section{Introduction-Infectious Disease Surveillance in India}

The emergence and re-emergence of infectious diseases like plague, SARS, H1N1, avian influenza and most recently the outbreak of Ebola and ZIKA viruses highlight the importance and urgent need for rapid outbreak detection and early warning. With the continuous acceleration and expansion of international trade, further globalization and amplified travelling, the spread of infectious diseases beyond national borders is faster than ever [1]. Infectious disease outbreaks can amplify conflicts as observed with the recent outbreak of Ebola in West Africa that lead to civil unrest, suspicion and 
aggression towards health workers [2]. Global public health surveillance for early identification and prevention of emerging and re-emerging infectious diseases has been recognized as an essential component of international collaboration and has also led to the development and further revision of the International Health Regulations (IHRs) of the U.N. World Health Organization (WHO) [3]. The revised IHRs of 2005 bind all 194 WHO member countries to provide sufficient and adequate trained human resources, money and material to implement the regulations for limiting the spread of public health emergencies of international concern [4,5]. This requires the reporting of health emergencies with potential of international concern even if the disease cause is unknown [6]. The 68th World Health Assembly (2015) re-emphasized the need for nondelayed IHR 2005 implementation and the respective public health capacity-building as a continuous process that is integrated in and linked with the health system development to ensure lasting and sustainable solutions [7].

Infectious disease surveillance and in particular the timely detection and early warning of disease outbreaks remain a complex challenge for the health authorities in India, despite considerable efforts to invest in an Integrated Disease Surveillance Programme (IDSP) [8]. Public health surveillance still lacks coordination as well as resources and adequate structure. Accordingly, the availability of reliable, comprehensive and timely health information is still insufficient for early warning of infectious diseases in India [9]. Health care responsibilities are shared among the federal (centre) and the respective state governments, which exhibit often divergent and sometimes competing political and developmental agendas. The control of outbreaks of infectious diseases lies with the (central) Union Ministry of Health but depends on cooperation and collaboration within the federal system [10]. Furthermore, available health data to assess the infectious disease burden in India is incomplete and erratic as reporting falls under the administration and oversight of various states, districts and further administrative units. Vaccine-preventable infectious diseases have been given priority through centrally-implemented (national) health programmes while other infectious diseases are not systematically controlled [11].

To some extent, gaps in monitoring infectious disease are the direct result of the current design of the Indian health system. The health system is very fragmented both administratively and thematically [12]. In recent decades there has been a bias towards technologically-driven advanced medical care which mostly serves the urban elite and middle class. Further, India is suffering from an inadequate and often dysfunctional public health infrastructure that limits and hinders prevention and control of infectious diseases in all strata of the Indian society [11,13]. Preventable infections are still a major cause of disabilities, morbidity and mortality in India [14] and structures to work towards such preventions, including surveillance and early warning systems, are not up to the task.

In this paper we take inventory of the needs and challenges for surveillance and early warning within the complex framework of public health surveillance in India. The aim of our study is to assess whether routinely-collected health information data from emergency medical services (EMS) can improve sustainable infectious disease surveillance and early warning capacity. We reflect on possible integration of EMS data for early warning of infectious disease outbreaks into existing government structure. Our argument starts from a critical reflection on the need of enhancing surveillance capacity in India. Drawing from a review of government documents, expert interviews and literature, we further reflect on the government's framing of missing elements and structural difficulties for infectious disease surveillance in India. We will argue that immediate improvement of the surveillance capacity could be achieved by including syndromic surveillance capacity based on routine health system data. To support our argument, we present and discuss a case study in the context of enhancing and supplementing the existing concepts for public health surveillance. We conclude with some recommendations to aid improving India's early warning capacities.

\section{Materials and Methods}

The research employs a mixed method approach including a series of semi-structured interviews with key informants in the field of disease surveillance in India and fever data analysis of the operating EMS dispatch system in the state of Andra Pradesh, India. The study follows a grounded theory 
concept using purposive sampling [15]. The research is structured into four phases: (I) the needs assessment of syndromic surveillance in India; (II) the conceptualization and design of a case study; (III) the test and evaluation of the concept in the case study; and (IV) the subsequent empirical rooted integration analysis.

(I) The needs assessment survey is based on a set of qualitative methods: (i) a review of government documents and related literature, (ii) 25 in-depth and semi-structured face-to-face interviews with stakeholders at different levels of the reporting network. Interviewees were recruited from responsible (health) administrators, EMS medical supervisors and dispatch managers, health professionals and surveillance experts, statisticians and consultants at state level (including the IDSP state surveillance units), district level and municipal level. The guiding interview questions are listed in Table 1. The interviews lasted on average $50 \mathrm{~min}$. The interviewees were asked for consent and were informed about the study in general before the interview took place. During the interview notes and quotes were taken and the interview was transcribed within $24 \mathrm{~h}$ after the interview took place. (iii) Field visits at selected health centres, hospitals and dispatch centres in undivided Andhra Pradesh, Maharashtra and Madhya Pradesh.

Table 1. Guiding interview questions for needs assessment survey.

\begin{tabular}{l} 
Interview Questions \\
\hline (1) How is the infectious disease surveillance system established in India? \\
(2) What are the major challenges of the infectious disease surveillance system? \\
(3) What where the major challenges during the most recent outbreak? \\
(4) How is the Integrated Disease Surveillance Programme (IDSP) implemented? \\
(5) What are the challenges of the IDSP? \\
(6) How is the communication between urban-district-state governments? \\
(7) Interlinkages of the central Health Programs to share data? \\
\hline
\end{tabular}

(II) For conceptualization and subsequent design of the case study, the System for Early warning based on Emergency Data (SEED), an interdisciplinary expert workshop bringing together professionals from the EMS syndromic surveillance project SIDARTHa [16,17], Indian surveillance system and EMS experts was organized. A participatory approach was used to jointly develop a framework adapted to the Indian surveillance needs for using EMS routinely collected data for infectious disease surveillance and early warning of outbreaks. A feasibility analysis of EMS data for infectious disease detection underlying the case studies was undertaken [18]. The selection of districts for the case studies was based on stratified system random sampling. The three districts (Sikakulam, Anantapur, Guntur) were selected based on the level of infant mortality rate, female literacy, urbanization, proportion of reported cases of fever/infections emergencies and proportion of the scheduled caste and tribes population to ensure a representative sampling. For this paper the district of Guntur was selected as a case in point to demonstrate proof of principle.

(III) The case study was performed for one selected district of undivided Andhra Pradesh in collaboration with GVK Emergency Management and Research Institute (GVK EMRI). GVK EMRI is the sole provider for emergency medical services in Andhra Pradesh under an exclusive state contract (PPP). The analysis was both retrospectively and prospectively undertaken using the symptom of Acute Undifferentiated Fever (AUF) to test the potential of early space-time identification of dengue outbreaks within the district. For the cluster detection of AUF, a space-time permutation model within the SaTScan software $[19,20]$ was used. For the interpolation of the cases per $\mathrm{km}^{2}$, a kernel density estimation using the CrimeStat IV software was used. The results were then imported for visualization in Quantum Geographic Information System (QGIS) [21]. For the verification of the detected outbreaks, local newspapers and additional medical records (where available) were consulted. 
(IV) The integration analysis was based on (i) the review of government documents on early warning and surveillance capacities, and (ii) 10 in-depth and semi-structured interviews with experts linked to the Integrated Disease Surveillance Project (IDSP) and experts in the field of public health surveillance and EMS in India. The analysis of the interviews followed the same approach as listed in the needs assessment methodology section. The focus here was on identifying possible integration opportunities of emergency medical service data for early warning of infectious diseases into the existing Indian surveillance and health reporting system. The guiding interview questions are outlined in Table 2.

Table 2. Guiding interview questions for System for Early warning based on Emergency Data (SEED) integration analysis into IDSP.

Interview Questions

(1) What is the aim of the IDSP?

(2) How is the IDSP implemented?

(3) What are the challenges of the IDSP?

(4) How is the communication between municipalities and the district?

(5) Are their interlinkages between the central health programs?

(6) What are the urgent needs in regards to public health surveillance?

(7) How is the early warning capacity regarding infectious disease surveillance?

(8) How is the data flow of IDSP?

(9) Where is the GVK EMRI EMS data captured and processed?

(10) What is the timeliness of EMS data in the GVK EMRI dispatch system (availability for analysis)?

Ethical clearance of this study was granted by the ethical committee from GVK Emergency Management Research Institute within the SEED project number: INDO/TRC/612/09-IHD.

\section{Results}

\subsection{Needs Assessment}

Over a decade ago, the National Commission on Macroeconomics and Health summarized the needs of the Indian public health (surveillance) system as follows: India urgently needs to "establish good surveillance system and develop validated data ( . . ) the data gaps need to be bridged, high priority accorded to operational research and adequate resources allocated" [22]. Also, the "evidence emphasizes the need to strengthen programme monitoring and supervision of implementation at all levels" [22].

India as a state party to the IHR 2005 is expected to develop capacities to detect, assess, notify and respond within its national borders to public health emergencies of international concern as of 2007. Even though many new initiatives and programmes have been developed and notable achievements in relation to infectious diseases surveillance have been accomplished, India is still struggling with early outbreak detection and warning, controlling and monitoring the spread of infectious diseases [11]. India had requested a two-year extension to implement IHR core capacities till end of 2016 [23] and as a consequence the state of implementation for India was not listed in the global status report to the 68th World Health Assembly.

The most common strategy of the various Indian governments for developing the health system and providing services to the continuously growing population is the implementation of vertical (national) health programs with only few programs dedicated to infectious diseases. Diseases that are not covered under the vertical (disease-specific) programmes are largely neglected and usually lack formal and comprehensive monitoring and control in most regions. There have been several attempts to improve the surveillance system in India and all national programs have also surveillance components built into the program structure. The former National Institute of Communicable Diseases (NICD) has 
been upgraded to be the Indian National Centre for Disease Control (NCDC) and to serve as a centre of excellence with responsibilities including rapid outbreak response and laboratory surveillance [24]. However, India has witnessed many large outbreaks of both emerging and re-emerging infections. Despite the aforementioned increased efforts, interviewed experts agree that the surveillance system is still not effective enough to assure the desired results.

Reasons for the limited success of the Indian public health surveillance system are manifold: the number of parallel systems under various vertical health programs, the lack of non-communicable diseases surveillance, limited inclusion and control of the growing number of private providers in the health sector, a rather scattered and inadequate laboratory network and last but not least the inadequate use of existing routine data for surveillance activities [25]. An adequate use of information technology for data collection, analysis and transmission is still missing and training and capacity building in quality data collection, as well as competence in processing analyzing and interpretation, needs further attention. The interviews revealed the persistence of this sectoral thinking in "silos" due to administrative and financial structures and responsibilities. As one consequence of the lack of a nationwide Health Information System (HIS), a general disregard of the implementation of a sufficient public health surveillance system seems to prevail [25]. The major challenges of public health surveillance lie in linking information from the several separately-operating health systems and in bridging the knowledge and information gaps among the various programs and stakeholders. According to the last report of a High Level Expert Group on Health (HLEG) established by the Planning Commission of India, there is an urgent need to restructure, integrate and establish a reliable health information system in India [26]. The HLEG as well as interviewed key informants recommended the development of a national health information network based on uniform standards that also ensures inter-operability among all stakeholders in the healthcare sector [26-28].

Disease surveillance in India is further affected by organizational and operational deficiencies. For many regions data is still collected and collated on paper, which results in various operational issues like duplication of efforts, inaccuracies in data collection, delays in detection of disease outbreaks, delayed response times for medical intervention and non-detection of many cases due to inaccurate or incomplete data. In short, all crucial components of an informed and evidence-based decision-making in public health are negatively affected by the deficiencies and shortcomings of current disease surveillance in India [7,28-30].

The draft of the National Health Policy 2015 states that the Indian national health programmes that address communicable diseases and that constitute the backbone of the public health system currently represent less than $6 \%$ of overall morbidity cases and cover just about $25 \%$ of all communicable diseases [31]. As a consequence, the policy draft calls for building up the necessary capacities to effectively respond to local outbreaks before they grow into larger epidemics. For building these and other required health system capacities, the draft National Health Policy 2015 suggests engagement with various stakeholders and relevant actors for inclusive partnerships, as the government alone cannot undertake the endeavour to provide health care for all [31]. Currently, three-quarters of the healthcare spending in India is paid privately [32]. Private-public-partnerships (PPP) are currently the preferential model for organizing the provision of EMS in India through exclusive or non-exclusive state contracts. High-performance EMS providers constitute highly-qualified potential partners for public health surveillance also as they routinely collect and manage data for operational and quality assurance purposes that can also be used for surveillance.

For rapid responses, containment and warning in case of an infectious disease outbreak, the responsible health authorities need the ability to quickly assess the actual impact and the potential risks. The effectiveness of this ability is determined by the availability of sound and up-to-date health information on infectious diseases. In response to these challenges the Government of India has initiated and partially implemented the Integrated Disease Surveillance Programme (IDSP). Its intention is to detect early warning signals of disease outbreaks and coordinate responses accordingly $[8,33]$. The IDSP is a decentralized, state-based surveillance system for a range of diseases 
that are of public health importance. The health data are collected at various entry points and are submitted to the district level. Reporting from the District Surveillance Unit (DSU) to the State Surveillance Unit (SSU) and the Central Surveillance Units (CSU) is done weekly via the Internet using the central IDSP portal. The IDSP weekly report includes three different standard reporting forms reflecting the range of specificity of the data. Interviewed experts agree that, so far, IDSP has not been able to achieve effective integration of interventions and strategies for public health. Involvement of the private sector (general practitioners, clinics, private labs, etc.) has been attempted in only a few regions and with limited success. The coverage of districts remains patchy, leading to wide gaps in ownership, resources, and reporting quality. According to the experts interviewed, one positive way to further strengthen the IDSP, in particular the early warning component, is the use of additional data sources for outbreak detection, including, for instance, EMS data.

In particular for countries where the disease reporting and surveillance system is still weak or underdeveloped, syndromic surveillance could be used as a supplementing system or even an alternative system, provided that routinely-collected electronic health data is available and can be processed in a timely manner. For our study we followed the definition of syndromic surveillance by the TripleS Project: "A real-time (or near-real time) collection, analysis, interpretation, and dissemination of health-related data to enable the early identification of the impact (or absence of impact) of potential human or veterinary public health threats that require effective public health action. Syndromic surveillance is based not on the laboratory-confirmed diagnosis of a disease but on non-specific health indicators including signs, symptoms as well as proxy measures (e.g., Absenteeism, drug sales, animal production collapse) that constitute a provisional diagnosis (or "syndrome"). The data are usually collected for purposes other than surveillance and, where possible, are automatically generated so as not to impose an additional burden on the data providers. This surveillance tends to be non-specific yet sensitive and rapid, and can augment and complement the information provided by traditional test-based surveillance systems" [34].

Further to lab-diagnosed disease reporting, pre-diagnosed syndromic surveillance (symptoms like fever, cough, etc.) can be beneficial in particular for early outbreak detection in the Indian context. With the increased availability of routinely-collected electronic health data in India, the possibility of automated disease surveillance and outbreak reporting is increasing.

India is regularly affected by dengue fever, malaria, Chikungunya and tuberculosis [35]. Nonspecific high fever is a common complaint that is often associated with the aforementioned diseases [18]. As certified government or government-contracted laboratories are limited, confirmation for clinical samples is often a challenge and thus underreporting of cases is the norm. Interviewees from various governmental departments and levels expressed the opinion that official figures represent just the tip of an iceberg of dengue fever cases. The government reporting only includes cases of dengue that were diagnosed at public hospitals and/or that had been confirmed by government-recognized laboratories. With only limited health data for dengue fever cases collected, any cost estimation for the social and economic burden of dengue fever in India is limited [36,37]. Furthermore, interviewed experts estimate the share between the public and private healthcare sector in India to be $30 \%$ and $70 \%$ of patients, respectively. The data use, accessibility and availability for infectious diseases reporting by the Indian government are mainly within the sphere of public data. This leads to serious underreporting of infectious diseases, a concern expressed in interviews with experts in disease surveillance.

To enhance the surveillance and public health reporting structure of infectious diseases, a comprehensive integrated system is needed, one in which the various routinely-collected health information data of the Indian health system are collated and continuously analyzed. Often in the Indian healthcare system, data are generated but subsequently are not transformed into useful information [27] or, as one expert phrases it: "data stay isolated without added meaning". With the rising triple burden of disease in India, consisting of infectious diseases, rapidly rising chronic (non-communicable diseases) and emergence of new pathogens causing epidemics and pandemics [6], 
strong surveillance competence is crucial to better manage scarce resources and avoid further spread of infectious diseases.

\subsection{System for Early Warning Based on Emergency Data (SEED)}

Having established the need for an effective heath data surveillance infrastructure including interoperability of all stakeholders in the healthcare sector, in the remaining part of this paper we move towards the demonstration of such an infrastructure, namely the use of routine data from the EMS services to detect fever outbreaks. At the same time, we further explore the potential of integrating EMS data into the IDSP.

The pilot project SEED, co-funded by the Indian Council for Medical Research and the German Ministry of Research and Education, was conceptualized and based on earlier experiences from the EMS syndromic surveillance project SIDARTHa [16,17,38,39]. The original project structure is shown in Figure 1. The starting point for the project was the assumption that calls from emergency medical services are routinely collected in electronic formats and can thus be analyzed and used for symptom/syndromic surveillance.

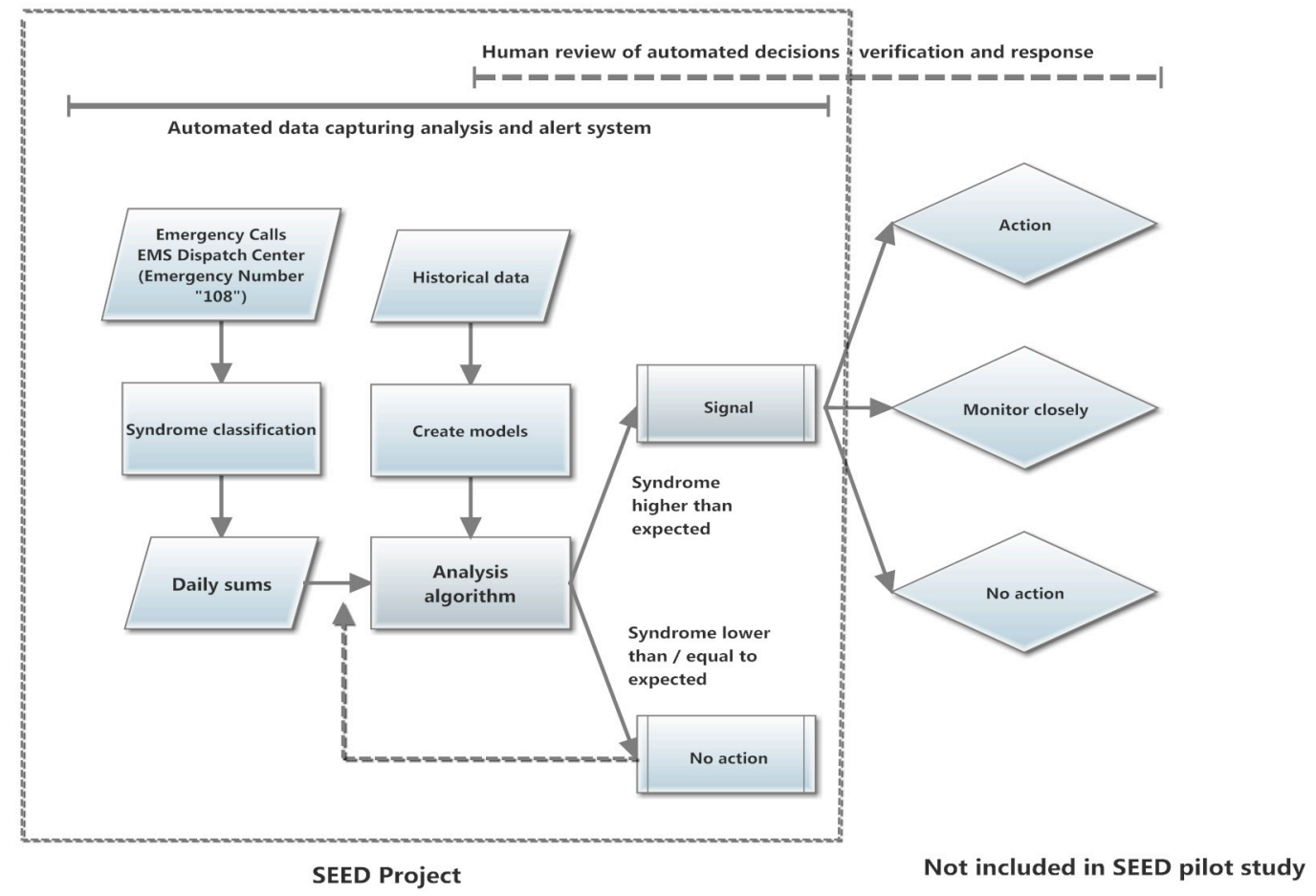

Figure 1. System for early warning using emergency data concept and data flow chart. Source: modified after Buehler, Hopkins, Overhage, Sosin, and Van Tong [40].

Figure 1 shows the process by which the routine EMS data from GVK EMRI used for the syndromic surveillance approach is generated automatically through incoming calls via the "108" universal emergency telephone number and captured at the dispatch centre in Hyderabad. All incoming calls are systematically assessed following standard medical protocols and then classified using a chief complaint-based approach. Using historical data from the system and local/regional spatial-temporal baselines, forecast algorithms calculate thresholds for syndromes on a continuously-updated basis. The thresholds represent the expected demand that would occur under "normal" conditions on a respective day and a respective time. The SEED compares the actual demand with the expected demand (forecasting) for a certain time and region (baseline) and issues an alert if the predefined thresholds are exceeded. Not included in the study are any actions/responses taken by authorities. 
The SEED system was tested at headquarters of the EMS provider GVK EMRI in Secunderabad, Andhra Pradesh. Technical results on fever cluster detection using EMS data and ARIMA models for forecasting needs in emergency-data-based syndromic surveillance, were published as part of the SEED [18,41,42]. The GVK EMRI EMS system is able to deliver routinely-collected health information (for purposes other than surveillance) in a comprehensive and cross-institutional manner [43].

\subsection{Test Phase of System for Early Warning Using Emergency Data/Case Study for Fever Surveillance in Guntur District, Andhra Pradesh, India}

In many parts of India, fever outbreaks are difficult to detect. Often the earliest acknowledgment of an outbreak is reported by the local newspapers, as surveillance systems are fragile and lacking in early warning capacity, according to key informants. Knowing where the outbreak occurs is the first step to develop a targeted response (vector control) to contain the outbreak. The case presents an example of the use of EMS data within the SEED approach to detect fever clusters at an early stage to enhance the early warning capacity. We tested retrospectively and prospectively cluster detection of AUF comprising space-time variation using open-source (freely available) systems like SaTScan, CrimeStat IV and QGIS to show the cost effectiveness and minimize cost implementation barriers for Guntur district in Andhra Pradesh. Data were obtained from the state emergency dispatch centre in Hyderabad. The emergency call data with the main complaint AUF-defined as any febrile illness lasting for $\leq 14$ days-were captured in the emergency dispatch centre using computer telephone integration technology (Figure 1) [18]. The data were available in Excel 2007 format and were already anonymized for privacy and confidentiality. The following call information was available: Date, district, mandal (smallest administrative unit covering populations $\leq 50,000$ ), village, age, sex, economic status, social status, time of emergency call, arrival time of the emergency vehicle and chief complaint. The data in Guntur district were available from 1 January to 30 September 2010 and consisted of 1595 fever emergency calls. Newspapers reported outbreaks of viral fever in September 2010. More than 1000 persons were suffering from fever in mosquito-prone Peddaparimi village [44,45]. Based on these newspaper reports, a cluster of fever emergency calls was expected between 5 September and 20 September 2010 from the EMS data.

\subsubsection{Retrospective Spatio-Temporal Fever Cluster Analysis}

The aim of the retrospective analysis of AUF is to analyze the data usefulness for fever cluster surveillance. Using a retrospective analysis allowed us to verify the outbreak through other data sources. For the retrospective space-time analysis, we used the space-time permutation model in SaTScan. The input data for this model consisted of the village coordinates and date of each fever emergency call. In this study, we set the maximum cluster size to not exceed $15 \mathrm{~km}$ in radius and 14 days in length. The results of the retrospective analysis of EMS AUF data detected three statistically significant clusters from January to September 2010. The most significant cluster $(p<0.001)$ occurred from 17 September to 27 September 2010 in Peddaparimi and Neerukonda village (Figure 2). This cluster consisted of 25 fever emergency calls and had a spatial radius of $2.70 \mathrm{~km}$. 


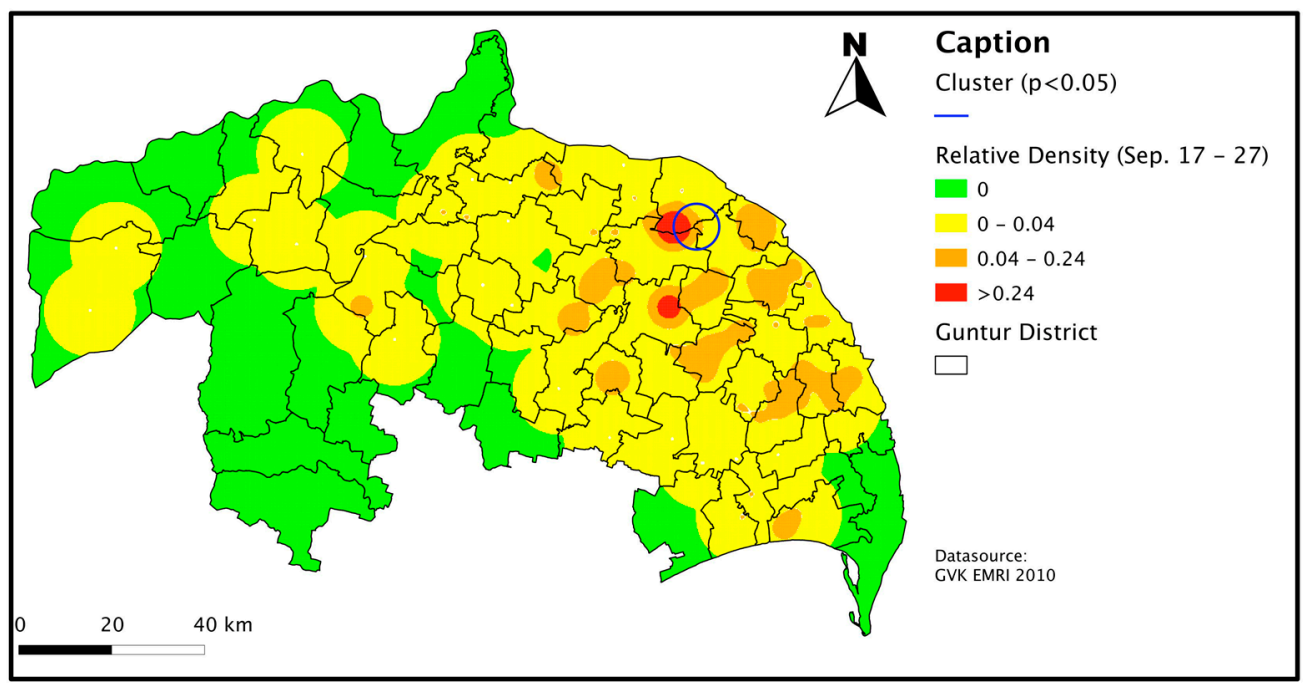

Figure 2. Results of the retrospective spatio-temporal analysis of Acute Undifferentiated Fever (AUF) in Guntur District, Andhra Pradesh, India in September 2010.

\subsubsection{Prospective Spatio-Temporal Cluster Analysis}

For timely surveillance near real-time data processing is crucial. To simulate SaTScans usage in a real-time syndromic surveillance system, a prospective analysis was performed for September 2010. In this simulation, new data was available every day from 1 September to 30 September 2010. A prospective spatio-temporal analysis was performed every day using the space-time permutation model in the SaTScan software. The maximum spatial radius size was defined to not exceed $15 \mathrm{~km}$. The maximum temporal scanning window was set to not include more than 14 days. The first significant cluster could be detected in Pedaparimi village in 3 September (week 1), consisting of five cases (Figure 3).

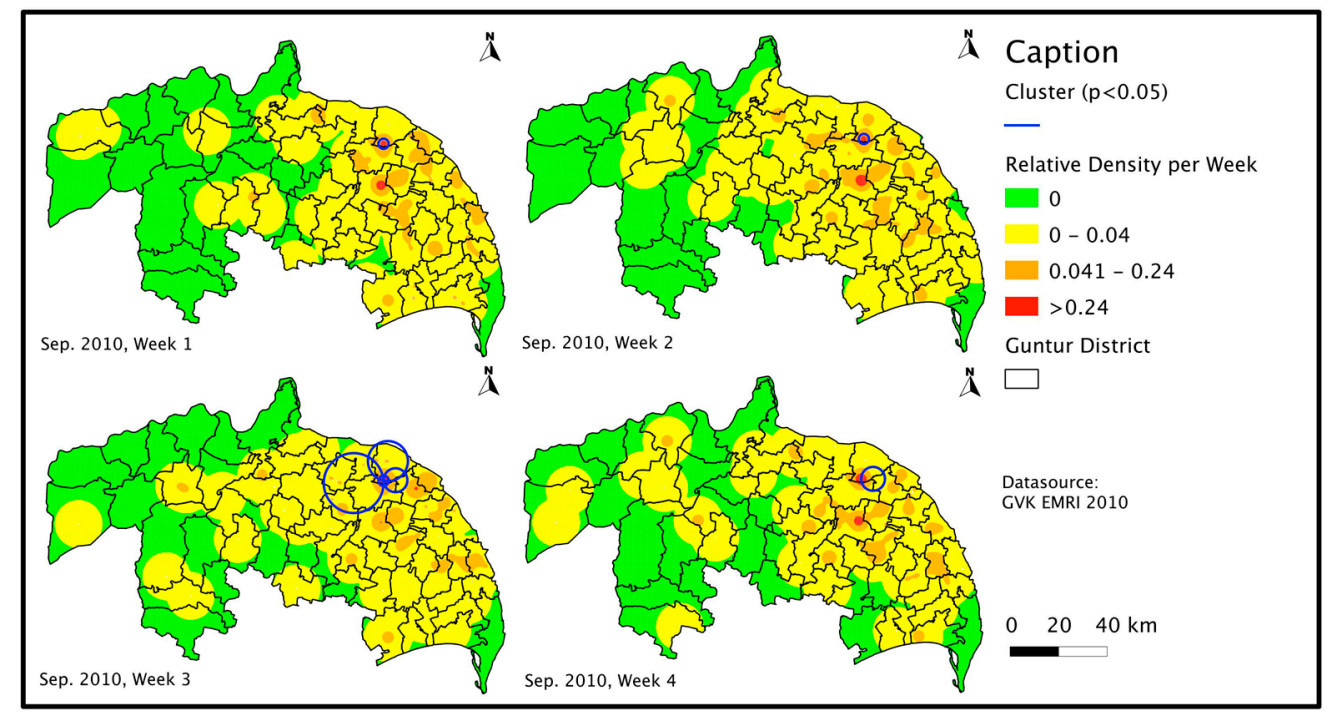

Figure 3. Results of the prospective spatio-temporal analysis of Acute Undifferentiated Fever (AUF) in Guntur District, Andhra Pradesh, India in September 2010.

At that time, the number of cases increased day by day and the same village could be detected as a significant cluster every day until 16 September. In the third week, SaTScan detected several clusters which all included Pedaparimi village. On 17 September, the cluster increased in spatial 
size and spread westwards of Pedaparimi village. On 18 September, no significant cluster could be observed. On 19 September the cluster spread northwards of Pedaparimi. Between 20 September and 30 September, the same cluster could be detected every day, consisting of Pedaparimi village and Neerukonda.

The prospective analysis provided an AUF cluster alarm already on 3 September, two days before the newspaper reported an increase of viral fevers and 17 days before the news outlets reported laboratory-confirmed cases of Dengue-fever. The AUF outbreak could be detected by applying SaTScan software and the complementary QGIS. It was evident that by using EMS data the AUF outbreak could be detected earlier than from any other source, including the newspapers, for early warning in the region.

\subsection{System of Early Warning Using Emergency Data Potential Integration Analysis into Integrated Disease Surveillance Program}

For the analysis, we chose to focus on the possibility of integrating the syndromic health information, generated on the basis of data from GVK-EMRI, at the level of state operations into the existing health information systems. The empirical rooted analysis is based on expert interviews with EMS and IDSP key informants and document analysis. An official from the Ministry of Health and Family Welfare mentioned that "Infectious diseases that are not covered in the national health programmes are neglected". Integration of vertical health programs and their coordination and monitoring with the local health care system is desired to efficiently reduce the infection disease burden. IDSP officials and EMS experts stressed that "Symptom based disease monitoring is not sufficiently used under the IDSP". As the IDSP is a state-based national health program, the structure follows a clear hierarchy of responsibilities with a state surveillance unit and the ministry of Health and Family Welfare at the state level [8]. The GVK EMRI EMS electronic health information gathered at the state dispatch centre is also already at state level but available in any relevant geographical resolution. The proposed information access for the Government of India can be established at the state surveillance unit as shown in Figure 4.

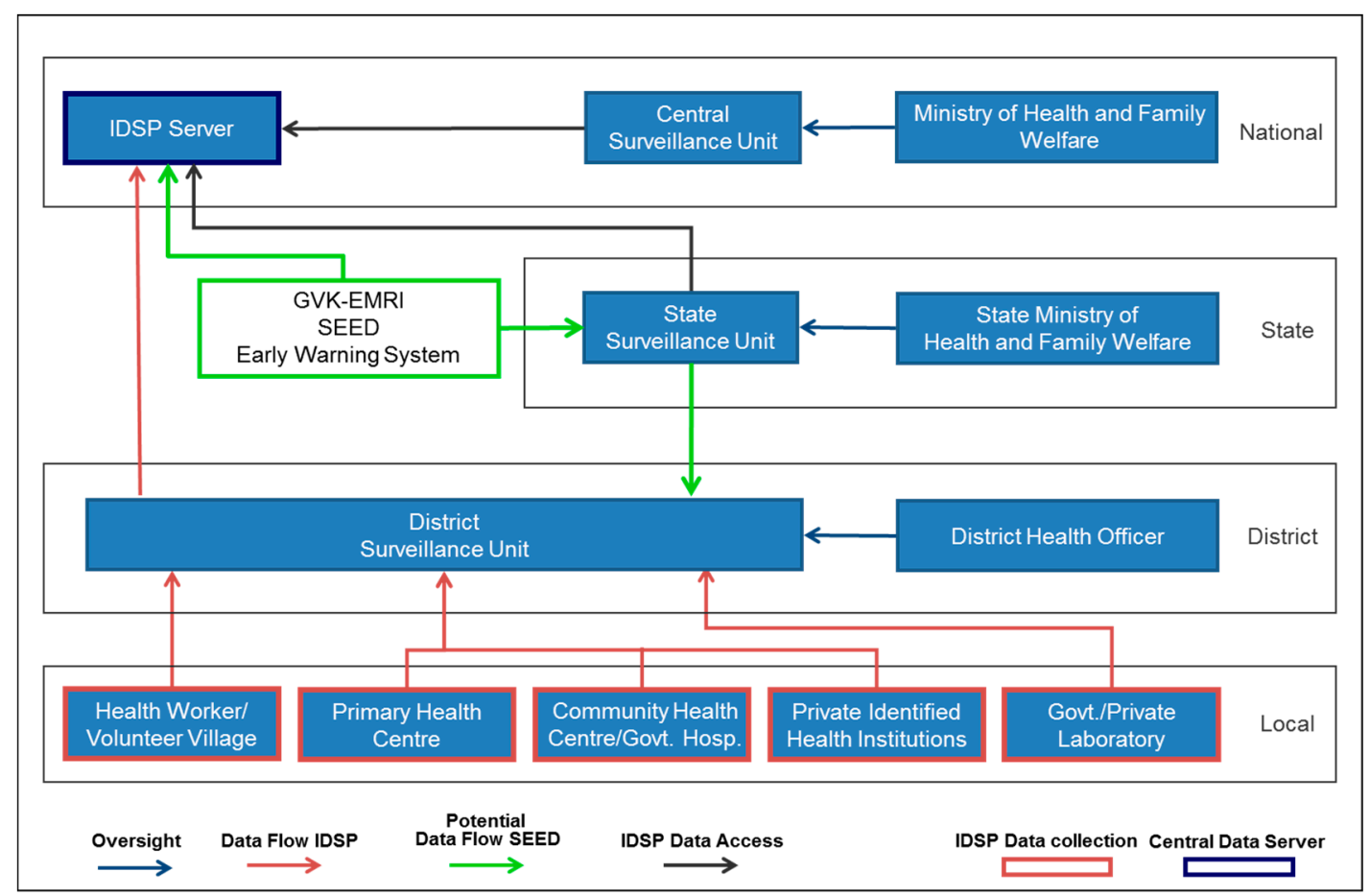

Figure 4. Integration of Emergency Medical Service data-based surveillance information into existing Integrated Disease Surveillance Programme infrastructure. 
In case of an alarm the surveillance system at GVK EMRI would assess and review to exclude false positives that can be explained by EMS systems knowledge and then inform the state surveillance unit. The data passed on would include the outbreak location and the symptoms/syndromes. The state surveillance unit will compare their own information to the SEED-generated information and decide on intervention procedures.

The benefit of incorporating the GVK EMRI outbreak detection/surveillance information into the existing IDSP structure is an additional data source (untouched so far) which can lead to even earlier and more precise future outbreak detection. Our aim is that SEED will contribute to the already existing IDSP infrastructure to enhance the current surveillance system. As shown in Figure 4, in case of an alarm indicating a potential outbreak an immediate link could be established to the state surveillance unit and ministry of health of the respected state. As the GVK EMRI operating EMS system is a public-private partnership with the infrastructure and data owned by the respective state government, the use of the data by the state government would benefit the existing early warning capacity in a very cost-efficient way. While there would be some costs involved in establishing the SEED at GVK EMRI and maintaining such a system, the costs are limited compared to other systems, since the data is generated anyway.

\section{Discussion}

Although "Importance of strengthening public health surveillance to provide early warning" is a repeated recommendation during the last decades [46], in particular the early warning components of infectious disease surveillance are still underdeveloped [22]. Especially, the sustainability of surveillance systems is not sufficiently taken into account, as disease reporting systems tend to be developed in administrative silos [10]. Although the IDSP was intended to overcome fragmentation and serve as a bridge between the vertically-implemented health programs and enable India to meet the IHRs, it has fallen short of reaching this objective [27,31,47]. The need to build up a sustainable infectious disease surveillance system to detect disease outbreaks earlier and with clear geographic location is paramount to comply with the IHR requirements.

The GVK EMRI state operations database contains relevant and near real-time symptom/ syndromic health information which is continuously and routinely updated but is currently not linked to regional and national public health information systems. With the EMS system from GVK EMRI developing across India, new opportunities for health surveillance are appearing [43,48]. Although the service is still under development and the number of emergency ambulances will have to grow to a sufficient size, the service has established an important role for the first access to the health care system in the operating states. A fully integrated and operational SEED would allow for earlier detection of communicable health threats at the regional and state levels, including spatial-temporal analysis. In a case of an unusual event (with a potential health threat) like the Icelandic ash cloud over Europe in 2010, such a system could serve as a first and immediately-available surveillance tool for situational awareness [49].

The Indian government has enhanced its efforts through the implementation of the IDSP to demonstrate commitment to improve infectious disease surveillance in response to the requirements of the revised IHR [50]. During the period of the 12th Five Year Plan and the drafted national health policy [31], the National Centre for Disease Control (NCDC) aim is to strengthen the control of infectious diseases and to implement the IHR [51]. The IDSP was the designed instrument with the aim to build up a comprehensive surveillance system combining separately-functioning surveillance activities, including symptom-based reporting, under one umbrella [33]. EMS data was not considered as a potential data source for enhancing the early warning capacity of IDSP so far. The use of EMS data, if available in standardized electronic near real-time format, can enhance the existing surveillance infrastructure and do so very cost efficiently. GVK EMRI provides the contracted EMS services for the respective state governments, based on a public-private partnership. Consequently, data ownership for reporting is already with the government and can, if adjusted and implemented under the IDSP, 
be a valuable addition to the existing surveillance system. This proof of principle can be applied to the other 33 (disease) syndromes captured by GVK EMRI data and can be transferred to any other region or Indian state served by GVK EMRI or by similarly-equipped and -managed EMS systems.

India needs to "establish good surveillance system and develop validated data ( . . ) the data gaps need to be bridged, high priority accorded to operational research and adequate resources allocated" [22]. And the "evidence emphasizes the need to strengthen programme monitoring and supervision of implementation at all levels" [22]. Capacity building and leadership in data handling, analyzing and the value of health information through data demand and analytical use, can improve data management and ultimately provide sustainable health information for evidence-based healthcare planning. Further, the UN High-Level Group for the Modernization of Official Statistics (HLG-MOS) found that the lack of analytical and data skills are one of the greatest challenges, even more than the lack of data quality [52].

As health information in India still demonstrates significant reliability and validity problems, further investment in health data research, to further identify the underlying reasons and develop and enhance a framework for an integrated health information system, is needed [53].

\section{Conclusions}

Our reviews of the relevant government documents and expert interviews nearly unanimously identify the need for improved early warning of infectious diseases and limitation in health surveillance due to lack of sufficient and reliable health information.

The SEED model demonstrated the capability of emergency data to be used for early detection and monitoring of infectious disease outbreaks in India. The application of the space-time scan statistic and the prospective space-time scan statistic provided substantial possibilities for an earlier detection of outbreaks. The sharp increase in the number of cases and the long-lasting aberration from the predicted demand allowed for early detection of the fever outbreak using AUF as indicator. In our study, SEED was just manually tested to evaluate if such a system would be feasible and could provide reliable data. It has proven its potential to be set up as a fully-automated system running near real time which can cost-efficiently enhance early warning capacity. To our knowledge, the SEED is the first attempt in India to comprehensively conceptualize a near real time database for disease surveillance constructed on EMS data. When implemented, it would not replace but would supplement and enhance existing health surveillance systems in India.

This study further demonstrated both the need and potential of early warning capacity via enlisting the currently untapped (for surveillance) standardized data sources. The implementation of a sustainable, cost-effective intervention has the potential to improve infectious disease surveillance in the immediate future and help the government to appropriately prioritize timely interventions. The use of routinely-collected health data for syndromic surveillance is sowing the SEEDs for responsible and sustainable public health decision making.

Acknowledgments: The initial System for Early warning using Emergency Data (SEED) Project was co-funded by the German Federal Ministry of Education and Research (BMBF) and the Indian Council for Medical Research (ICMR) in the Field of Public Health 2009-2011 (Grant Agreement No. BMBF:IND08/005; ICMR:INDO/TRC/612/09-IHD). The authors thank Bart Penders and Anja Krumeich for input on the final draft.

Author Contributions: Eva Pilot wrote the first draft; Eva Pilot, Ramana Roa, Biranchi Jena and Thomas Krafft conceived and designed the study; Boris Kauhl performed the GIS analysis; Eva Pilot undertook the interviews and analyzed the data; Eva Pilot, Ramana Roa, Biranchi Jena, Thomas Krafft and GVS Murthy discussed and facilitated agreement in the end; all authors commented on and contributed to the draft and approved the final version.

Conflicts of Interest: The authors declare no conflicts of interest.

\section{References}

1. World Health Organization. The World Health Report 2007: A Safer Furture Global Public Health Security in the 21st Century; World Health Organization: Geneva, Switzerland, 2007. 
2. Bayntun, C.; Houlihan, C.; Edmunds, J. Ebola crisis: Beliefs and behaviours warrant urgent attention. Lancet 2014, 384, 1424. [CrossRef]

3. Nuttall, I. International Health Regulations (2005): Taking stock. Bull. WHO 2014, 92, 310. [CrossRef] [PubMed]

4. World Health Organization. International Health Regulations (2005), 2nd ed.; World Health Organization: Geneva, Switzerland, 2008.

5. Ijaz, K.; Kasowski, E.; Arthur, R.R.; Angulo, F.J.; Dowell, S.F. International Health Regulations-What Gets Measured Gets Done. Emerg. Infect. Dis. 2012, 18, 1054-1057. [CrossRef] [PubMed]

6. May, L.; Chretien, J.P.; Pavlin, J. Beyond traditional surveillance: Applying syndromic surveillance to developing settings-opportunities and challenges. BMC Public Health 2009, 9, 242. [CrossRef] [PubMed]

7. World Health Organization. Implementation of the International Health Regulations (2005). Responding to Public Health Emergencies; World Health Organization: Geneva, Switzerland, 2015.

8. Suresh, K. Integrated Diseases Surveillance Project (IDSP) Through a Consultant's Lens. Indian J. Public Health 2008, 52, 136-143. [PubMed]

9. Davies, S.E. The challenge to know and control: Disease outbreak surveillance and alerts in China and India. Glob. Public Health 2012, 7, 695-716. [CrossRef] [PubMed]

10. Flahault, A. Emerging infectious diseases: The example of the Indian Ocean chikungunya outbreak (2005-2006). Bull. L'Academie Natl. Med. 2007, 191, 113-124.

11. John, T.J.; Dandona, L.; Sharma, V.P.; Kakkar, M. Continuing challenge of infectious diseases in India. Lancet 2011, 377, 252-269. [CrossRef]

12. Patel, V.; Kumar, A.K.S.; Paul, V.K.; Rao, K.D.; Reddy, K.S. Universal health care in India: The time is right. Lancet 2011, 377, 448-449. [CrossRef]

13. Narain, J.P. Public Health Challenges in India: Seizing the Opportunities. Indian J. Community Med. 2016, 41, 85-88. [CrossRef] [PubMed]

14. Zaidi, A.K.M.; Awasthi, S.; deSilva, H.J. Burden of infectious diseases in South Asia. BMJ (Clin. Res. Ed.) 2004, 328, 811-815. [CrossRef] [PubMed]

15. Charmaz, K. Constructing Grounded Theory: A Practical Guide Through Qualitative Analysis; SAGE Publications: London, UK, 2006.

16. Riesgo, L.G.; Ziemann, A.; Rosenkoetter, N.; Krafft, T.; Fischer, M.; Lippert, F.; Vergeiner, G.; Gillet, J.B.; Meulemans, A.; Kraemer, A.; et al. Use of routinely collected emergency medical data for earlier detection of health threats in Europe: First evaluation results of the SIDARTHa syndromic surveillance system. Resuscitation 2010, 81, S7. [CrossRef]

17. Ziemann, A.; Riesgo, L.G.; Boris, K.; Schrell, S.; Rosenkötter, N.; Fischer, M.; Vergeiner, G.; Gillet, J.; Meulemans, A.; Krafft, T. Added value of routine emergency medical data for detecting clusters of acute gastrointestinal illness in Europe. Resuscitation 2012, 83, e30. [CrossRef]

18. Jena, B.; Murthy, M.P.; Ramanarao, G. Demand pattern of Medical Emergency Services for Infectious Diseases in Andhra Pradesh-A Geo spatial Temporal Analysis of Fever cases. Indian Emerg. J. 2010, V, 5-9.

19. Costa, M.A.; Kulldorff, M. Maximum linkage space-time permutation scan statistics for disease outbreak detection. Int. J. Health Geogr. 2014, 13, 20. [CrossRef] [PubMed]

20. SaTScan. SaTScan Software for the Spatial, Temporal, and Space-Time Scan Statistics. Available online: http:/ / www.satscan.org/ (accessed on 26 September 2016).

21. QGIS. QGIS A Free and Open Source Geographic Information System. 2016. Available online: http:/ / www.qgis.org/en/site/ (accessed on 26 September 2016).

22. National Commission on Macroeconomics and Health. Burden of Disease in India; National Commission on Macroeconomics and Health: New Delhi, India, 2005.

23. World Health Organization. Country Cooperation Strategy India; World Health Organization: Geneva, Switzerland, 2015.

24. Dikid, T.; Jain, S.K.; Sharma, A.; Kumar, A.; Narain, J.P. Emerging \& re-emerging infections in India: An overview. Indian J. Med. Res. 2013, 138, 19-31. [PubMed]

25. Bachani, D. Integration of disease surveillance in India: Current scenario and future perspective. Indian J. Public Health 2006, 50, 7-10. [PubMed]

26. High Level Expert Group for Universal Health Coveage. Executive Summary: High Level Expert Group Report on Universal Health Coverage for India; Planning Commission of India: New Delhi, India, 2011. 
27. Instituted by the Planning Commission of India. High Level Expert Group Report on Universal Health Coverage for India; Planning Commission of India: New Delhi, India, 2011.

28. Pilot, E.; Rao, R.; Jena, B.; Krafft, T. Emergency Medical Service (EMS) Data for Syndromic Surveillance in Andhra Pradesh, India. In Environment and Health in Urban Areas; Wang, W., Krafft, T., Rosenberg, M., Pilot, E., Eds.; China Environmental Press: Beijing, China, 2014.

29. Foreit, K.; Moreland, S.; LaFond, A. Data Demand and Information Use in the Health Sector Conceptual Framework; MEASURE Evaluation: Chapel Hill, NC, USA, 2006.

30. Moreland, S.; Misra, S.N.; Agarwal, S.; Gupta, R.B.; Harrison, T.; Agrawal, S.; Gupta, R.B.; Harrison, T. Data Use in the Indian Health Sector; MEASURE Evaluation: Chapel Hill, NC, USA, 2009.

31. Ministry of Health and Family Welfare. National Health Policy 2015 Draft; Ministry of Health and Family Welfare: New Delhi, India, 2014.

32. Balarajan, Y.; Selvaraj, S.; Subramanian, S.V. Health care and equity in India. Lancet 2011, 377, 505-515. [CrossRef]

33. NCDC; MoHFW; GoI. Integrated Disease Surveillance Programme (IDSP). Available online: http:/ / www. idsp.nic.in/ (accessed on 10 March 2015).

34. TripleS Project. Assessment of syndromic surveillance in Europe. Lancet 2011, 378, 1833-1834.

35. Seyler, T.; Hutin, Y.; Ramanchandran, V.; Ramakrishnan, R.; Manickam, P.; Murhekar, M. Estimating the burden of disease and the economic cost attributable to chikungunya, Andhra Pradesh, India, 2005-2006. Trans. R. Soc. Trop. Med. Hyg. 2010, 104, 133-138. [CrossRef] [PubMed]

36. Gore, M.M. Need for constant monitoring of dengue infections. Indian J. Med. Res. 2005, 121, 9-12. [PubMed]

37. Garg, P.; Nagpal, J.; Khairnar, P.; Seneviratne, S.L. Economic burden of dengue infections in India. Trans. $R$. Soc. Trop. Med. Hyg. 2008, 102, 570-577. [CrossRef] [PubMed]

38. Rosenkötter, N.; Ziemann, A.; Krafft, T.; Riesgo, L.G.; Vergeiner, G.; Brand, H. Non-infectious events under the International Health Regulations (2005) in Europe-A case for syndromic surveillance. J. Public Health Policy 2014, 35, 311-326. [CrossRef] [PubMed]

39. Ziemann, A.; Rosenkötter, N.; Riesgo, L.; Fischer, M.; Krämer, A.; Lippert, F.K.; Vergeiner, G.; Brand, H.; Krafft, T. Meeting the International Health Regulations (2005) surveillance core capacity requirements at the subnational level in Europe: The added value of syndromic surveillance. BMC Public Health 2015, 15, 107. [CrossRef] [PubMed]

40. Buehler, J.; Hopkins, R.S.; Overhage, J.M.; Sosin, D.M.; Van Tong, M.P.H. Framework for Evaluating Public Health Surveillance Systems for Early Detection of Outbreaks Recommendations from the CDC Working Group. MMWR. 2014, 53, 1-11.

41. Umar, N.S.; MNV, P.; Jena, B. Use of ARIMA models for forecasting needs in Emergency data based Syndromic Surveillance. Indian Emerg. J. 2010, V, 17-20.

42. Kauhl, B.; Pilot, E.; Rao, R.; Gruebner, O.; Schweikart, J.; Krafft, T. Estimating the spatial distribution of acute undifferentiated fever (AUF) and associated risk factors using emergency call data in India. A symptom-based approach for public health surveillance. Health Place 2015, 31, 111-119. [CrossRef] [PubMed]

43. Kumutha, J.; Rao, G.V.R.; Sridhar, B.N.; Vidyasagar, D. The GVK EMRI maternal and neonatal transport system in India: A mega plan for a mammoth problem. Semi. Fetal Neonatal Med. 2015, 20, 326-334. [CrossRef] [PubMed]

44. Express News Service. Health Emergency in Guntur District; Indian Express, 2010. Available online: http:/ / www.newindianexpress.com/states/andhra_pradesh/article297732.ece (accessed on 20 May 2007).

45. Ramagopal, S. Not an Emergency, But a Calamity in Guntur-The New Indian Express; Indian Express, 2010; Available online: http://www.newindianexpress.com/states/andhra_pradesh/article300929.ece (accessed on 1 October 2014).

46. Morse, S.S. Public health surveillance and infectious disease detection. Biosecur. bioterror. 2012, 10, 6-16. [CrossRef] [PubMed]

47. May, L.; Katz, R.L.; Test, E.; Baker, J. Applications of Syndromic Surveillance in Resource Poor Settings. World Med. Health Policy 2011, 3, 1-29. [CrossRef]

48. Garg, R.H. Who killed Rambhor?: The state of emergency medical services in India. J. Emerg. Trauma Shock. 2012, 5, 49-54. [CrossRef] [PubMed]

49. Brand, H.; Krafft, T. The icelandic ash cloud and other erupting health threats:what role for syndormic surveillance? Eur. J. Public Health 2010, 20, 367-368. [CrossRef] [PubMed] 
50. Kant, L.; Krishnan, S.K. Information and communication technology in disease surveillance, India: A case study. BMC Public Health 2010, 10, S11. [CrossRef] [PubMed]

51. Planning Commission Government of India. Twelfth Five Year Plan (2012-2017) Economic Sector Volume II; Planning Commission Government of India: New Delhi, India, 2013.

52. UNECE HLG-MOS. High-Level Group for the Modernisation of Official Statistics. 2016. Available online: http://www1.unece.org/stat/platform/display/hlgbas/High-Level+Group+for+the+ Modernisation+of+Official+Statistics (accessed on 1 January 2016).

53. Krafft, T.; Pilot, E.; Sarkar, B.K.; Ryland, P.; Reeves, L.; Singh, V. Research and Innovations Guiding Public Health Surveillance in the Twenty-first Century. In Transforming Public Health Surveillance; McNabb, S., Code, J., Ferland, L., Macwright, W., Okutani, S., Park, M., Ryland, P., Shakh, A., Singh, V., Conde, M., Eds.; Elsevier: Amsterdam, The Netherlands, 2015; pp. 388-397.

(C) 2017 by the authors. Licensee MDPI, Basel, Switzerland. This article is an open access article distributed under the terms and conditions of the Creative Commons Attribution (CC BY) license (http:/ / creativecommons.org/licenses/by/4.0/). 